\title{
Variación en el patrón de consumo de cannabinoides sintéticos de una paciente a lo largo de 2018
}

\section{Variation in the pattern of synthetic cannabinoid use by a female patient during 2018}

\author{
David Fabregat-Safont*, Carmen Ripoll**, Teresa Orengo***, Juan V. Sancho*, \\ FÉlix HernándeZ*, María Ibáñez*. \\ * Research Institute for Pesticides and Water. University Jaume I, Castellón. España. \\ ** Addictions Treatment Unit Campanar, Valencia. España. \\ *** Addictions Treatment Unit Grao-Clínico, Valencia. España.
}

$\mathrm{E}$ 1 consumo de cannabinoides sintéticos (CS) es, en la actualidad, objeto de gran preocupación. Una búsqueda rápida en la bibliografía muestra cómo estos compuestos han sido relacionados con numerosas intoxicaciones (Adams et al., 2017; Kusano et al., 2018). El Sistema Español de Alerta Temprana (SEAT) ha notificado la detección en 7 nuevos CS desde 2014 (Observatorio Español de las Drogas y las Adicciones, 2017). Además, se han reportado diversos CS presentes en herbal blends adquiridas en tiendas españolas (Ibáñez et al., 2013). No obstante, la detección del consumo de CS a través del análisis de orina no se puede realizar de forma directa ya que estas sustancias se metabolizan rápida y completamente, siendo necesario detectar sus principales metabolitos (Scheidweiler, Jarvis y Huestis, 2015).

En este trabajo se ha realizado el seguimiento de una consumidora de CS que acudía a una Unidad de Conductas Adictivas (UCA) de Valencia (España). Se analizaron muestras de orina de la paciente, así como hierbas y cigarros que se le requisaron. También se analizaron hierbas vendidas en la smart-shop donde se sospechaba que compraba la paciente. El cambio observado en el patrón de consumo se relacionó con la prohibición de ciertos cannabinoides en España (Ministerio de Sanidad Consumo y Bienestar Social, 2018).

Las muestras de orina y hierbas se recogieron en las UCAs participantes en el estudio, siguiendo el protocolo aprobado por el comité de ética local (Ref: DGNRI6 1424-10). La orina y las hierbas incautadas a la paciente se recogieron en la UCA donde recibe tratamiento, mientras que la segunda UCA incautó hierbas y cigarros a otros pacientes. Adicionalmente, se compraron hierbas en una conocida smart-shop de la localidad donde vive la paciente. El análisis de hierbas y orinas se llevó a cabo siguiendo la metodología descrita en bibliografía (Fabregat-Safont et al., 2017; Ibáñez et al., 2013).

Los análisis se realizaron mediante cromatografía líquida-espectrometría de masas de alta resolución. Se utilizó un cromatógrafo de líquidos Acquity I-Class (Waters, Mildford, MA, USA) acoplado a un sistema Vion IMS Qtof (Waters, Manchester, UK) usando ionización por electrospray.

Cuando la paciente acude a la UCA es una adolescente de 17 años que fue adoptada a los 6, procedente de los países del Este. Consulta por consumo de cannabis y acude por presión de los padres, ya que tiene nula conciencia del problema. Ha seguido tratamiento en psiquiatría infantil desde los 11 años por trastornos del comportamiento y retraso mental leve. La relación con los padres no es buena y, además, resultan poco eficaces en el manejo de la paciente. Ha tenido conductas autolesivas y problemas con Fiscalía de Menores sin consecuencias. Ha abandonado los estudios.

La paciente reconoció en las entrevistas realizadas antes del verano el consumo de cierta hierba llamada Hardcore (Figura 1A). Tras analizar este producto, se encontraron hasta $3 \mathrm{CS}$, todos pertenecientes a la misma familia: XLR11, UR-144 y, en menor proporción, el análogo $N$-pentil clorado del UR-144. Una vez conocida la composición del

Recibido: Julio 2019; Aceptado: Noviembre 2019.

Enviar correspondencia a:

María Ibáñez, Instituto Universitario de Plaguicidas y Aguas, Universitat Jaume I de Castelló.

E-mail: ibanezm@uji.es 
producto, se buscaron en la orina de la paciente los metabolitos descritos para el XLR-11 y UR-144 (Jang et al., 2016). En 2 de las 3 muestras de orina recogidas a la pa- ciente durante el primer semestre de 2018, se detectó el principal metabolito del XRL-11 (ácido $N$-pentanoico).
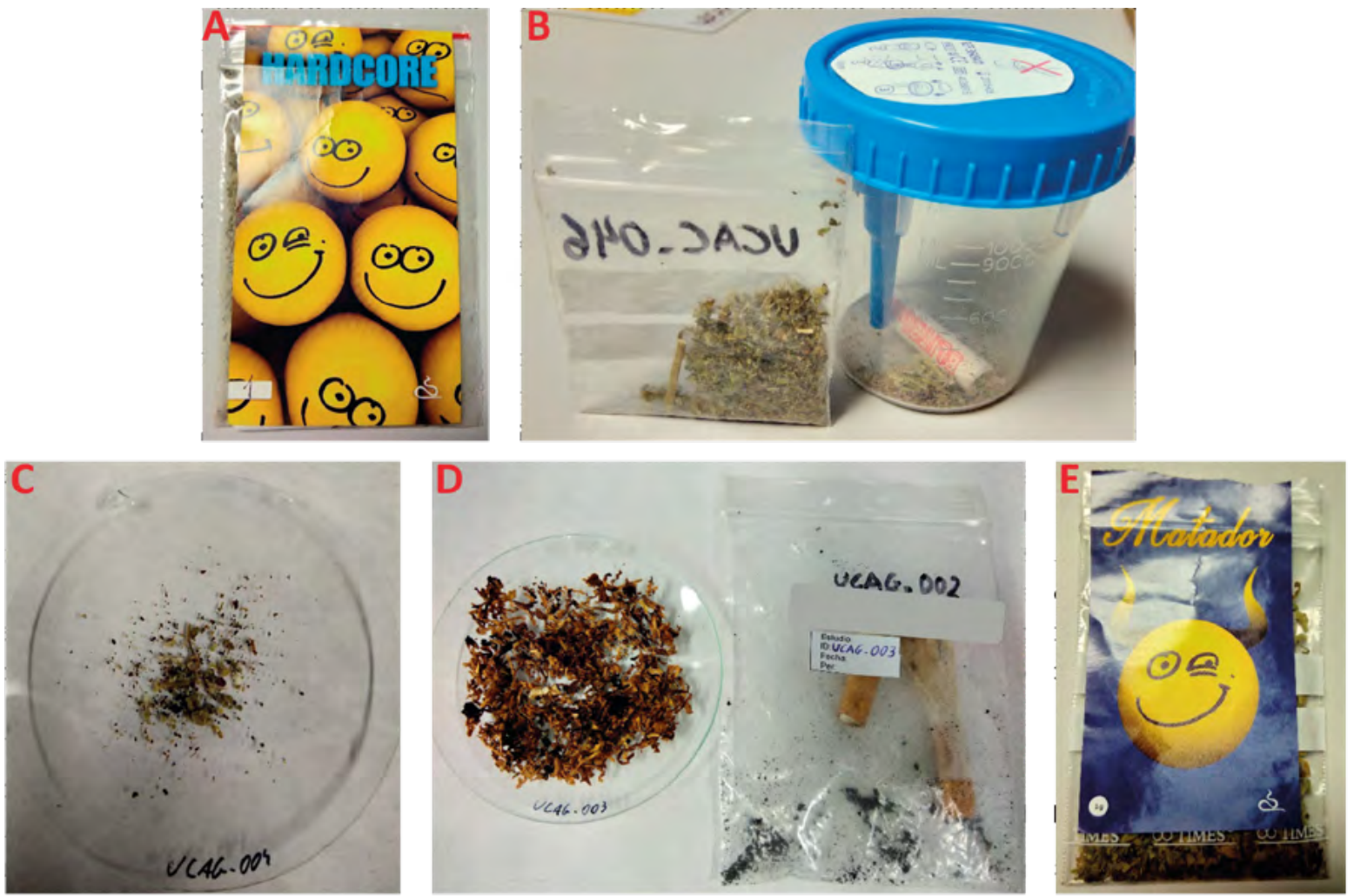

Figura 1. Muestras de consumo analizadas. A Hierba Hardcore que contiene XLR-11 y UR-144. B, C Hierbas incautadas en las UCAs que contienen 5F-ADB. D Cigarro incautado en la UCA que contiene 5F-ADB. E Hierba Matador que contiene 5F-ADB.

Sin embargo, en la orina recogida después del verano, ya no se encontró ningún metabolito del XLR-11. Así pues, o el sujeto había dejado de consumir CS, o habían cambiado sus hábitos de consumo. Una rápida búsqueda en la legislación española reveló que el XLR-11 había sido prohibido en el Boletín Oficial del Estado el 12 de julio de 2018, (Ministerio de Sanidad Consumo y Bienestar Social, 2018). Parecía lógico pensar que el sujeto hubiese cambiado sus patrones de consumo, aunque se desconocía que sustancia pudiera estar fumando en ese momento. Semanas después, se le incautaron diversas muestras de hierba (Figura 1B). Los análisis revelaron un nuevo y peligroso CS conocido como 5F-ADB o 5F-MDMB-PINACA. A este compuesto se le atribuye una elevada potencia (Banister et al., 2016) y ha sido relacionado con diversas muertes (Hasegawa et al., 2015; Kusano et al., 2018).

Una vez identificado este nuevo CS, se reprocesaron las muestras de orina buscando 3 metabolitos descritos para el 5F-ADB (Kusano et al., 2018). En 2 de las 6 muestras tomadas a la paciente tras el verano, se detectaron los tres metabolitos principales del 5F-ADB. Los resultados obtenidos sugieren que la prohibición del XLR-11 instigó a los fabri- cantes y/o vendedores de nuevas sustancias psicoactivas a cambiar este producto por otro que no estuviera legislado.

Unas semanas después, se recogió una tercera hierba (Figura 1C) y un cigarro (Figura 1D) en dos sujetos atendidos en la segunda UCA participante. Además, se adquirió una nueva herbal blend disponible en la smart-shop local (Matador, Figura 1E). En todas estas muestras se encontró 5F-ADB, sugiriendo que esta sustancia se empezó a consumir de forma frecuente en Valencia después del verano.

Nuestro trabajo ilustra cómo la prohibición de CS puede alterar sus patrones de consumo. Estos resultados sugieren que las tendencias de consumo de CS dependen en gran medida de la legislación vigente, de modo que cuando un cannabinoide se prohíbe, desaparece del mercado y se sustituye por un nuevo compuesto, que llega rápidamente a las calles.

\section{Reconocimientos}

Los autores agradecen la financiación recibida del Ministerio de Economía y Competitividad de España (Proyecto CTQ2015-65603-P) y por la Universitat Jaume I (Pro- 
yecto UJI-B2018-19). David Fabregat-Safont agradece al Ministerio de Educación, Cultura y Deporte de España su contrato predoctoral (Beca FPU15/02033).

\section{Conflicto de intereses}

Los autores declaran la inexistencia de conflictos de interés.

\section{Referencias}

Adams, A. J., Banister, S. D., Irizarry, L., Trecki, J., Schwartz, M. y Gerona, R. (2017). "Zombie” Outbreak Caused by the Synthetic Cannabinoid AMB-FUBINACA in New York. New England Journal of Medicine, 376, 235-242. doi:10.1056/NEJMoa1610300.

Banister, S. D., Longworth, M., Kevin, R., Sachdev, S., Santiago, M., Stuart, J., ... Kassiou, M. (2016). Pharmacology of Valinate and tert -Leucinate Synthetic Cannabinoids 5F-AMBICA, 5F-AMB, 5F-ADB, AMB-FUBINACA, MDMB-FUBINACA, MDMB-CHMICA, and Their Analogues. ACS Chemical Neuroscience, 7, 1241-1254. doi: 10.1021/acschemneuro.6b00137.

Fabregat-Safont, D., Barneo-Muñoz, M., Martinez-Garcia, F., Sancho, J. V., Hernández, F. y Ibáñez, M. (2017). Proposal of 5-methoxy-N-methyl-N-isopropyltryptamine consumption biomarkers through identification of in vivo metabolites from mice. Journal of Chromatography A, 1508. doi:10.1016/j.chroma.2017.06.010.

Hasegawa, K., Wurita, A., Minakata, K., Gonmori, K., Yamagishi, I., Nozawa, H., ... Suzuki, O. (2015). Identification and quantitation of 5-fluoro-ADB, one of the most dangerous synthetic cannabinoids, in the stomach contents and solid tissues of a human cadaver and in some herbal products. Forensic Toxicology, 33, 112-121. doi:10.1007/ s11419-014-0259-0.

Ibáñez, M., Bijlsma, L., van Nuijs, A. L. N., Sancho, J. V., Haro, G., Covaci, A. y Hernández, F. (2013). Quadrupole-time-of-flight mass spectrometry screening for synthetic cannabinoids in herbal blends. Journal of Mass Spectrometry, 48, 685-694. doi:10.1002/jms.3217.

Jang, M., Kim, I. S., Park, Y. N., Kim, J., Han, I., Baeck, S., ... Yoo, H. H. (2016). Determination of urinary metabolites of XLR-11 by liquid chromatography-quadrupole time-of-flight mass spectrometry. Analytical and Bioanalytical Chemistry, 408, 503-516. doi:10.1007/s00216015-9116-1.

Kusano, M., Zaitsu, K., Taki, K., Hisatsune, K., Nakajima, J., Moriyasu, T., ... Ishii, A. (2018). Fatal intoxication by $5 \mathrm{~F}-\mathrm{ADB}$ and diphenidine: Detection, quantification, and investigation of their main metabolic pathways in humans by LC/MS/MS and LC/Q-TOFMS. Drug Testing and Analysis, 10, 284-293. doi:10.1002/dta.2215.
Ministerio de Sanidad Consumo y Bienestar Social (2018). BOE-A-2018-10185. Boletín Oficial Del Estado, 175, 73005-73006.

Observatorio Español de las Drogas y las Adicciones (2017). INFORME 2017. Alcohol, tabaco y drogas ilegales en España. Observatorio Español de La Droga y Las Toxicomanias. Ministerio de Sanidad y Servicios Sociales.

Scheidweiler, K. B., Jarvis, M. J. Y. y Huestis, M. A. (2015). Nontargeted SWATH acquisition for identifying 47 synthetic cannabinoid metabolites in human urine by liquid chromatography-high-resolution tandem mass spectrometry. Analytical and Bioanalytical Chemistry, 407, 883-897. doi:10.1007/s00216-014-8118-8. 